\title{
Distributed Beamforming with Nodes Selection for Cognitive Radio Networks
}

\author{
X.Lian, H. Nikookar and L.P. Ligthart \\ Faculty of Electrical Engineering, Mathematics and Computer Science, \\ Microwave Sensing, Systems and Signals (MS3) Group, \\ Delft University of Technology, Mekelweg 4, 2628CD Delft, The Netherlands \\ xiaohualiantud@gmail.com,h.nikookar@tudelft.nl,L.P.Ligthart@tudelft.nl
}

Received September 2013; Accepted November 2013

Publication January 2014

\begin{abstract}
In order to collaboratively forward the Cognitive Radio (CR) signal to the Distant CR (DCR) users, we have introduced the Distributed Beamforming (DB) technique CR networks. However we face a practical difficulty of the extreme narrow main beam in the pattern generated by the DB method, when applying it to CR networks. To solve this problem, we propose a novel Nodes Selection (NS) method based on studies of the differences in beam width of a broadside array and an end-fire array. The proposed NS method selects those CR nodes, which are able to form a full size end-fire array and a reduced size broadside array. It chooses CR nodes located in the "belt" area along the direction of the DCR user. Simulation results of the average beampattern of our NS method show that the main beams are successfully directed towards the DCR users and are enlarged for practical applications in CR networks. What is more, for a CR network with a large physical size, our NS method can widen the main beam while maintaining sufficiently low sidelobe levels for CR transmission.
\end{abstract}

Keywords: Cognitive Radio (CR), Distributed Beamforming (DB), Collaborative Beamforming, Cognitive Radio network, Nodes Selection (NS).

Journal of Communication, Navigation, Sensing and Services, Vol. 1, 23-46.

doi: 10.13052/jconasense2246-2120.112

(c) 2014 River Publishers. All rights reserved. 


\section{Introduction}

Cognitive Radio (CR) is a promising solution to solve the problem of intensive usage of the natural radio resource, spectrum. It has been initially introduced by Joseph Mitola [1], who described how CR could enhance the flexibility of wireless services through a radio knowledge representation language. CR provides various solutions to accommodate this spectrum to be used by unlicensed wireless devices without disrupting the communications of the Primary Users (PUs) of the spectrum [2]. CR capabilities can also be exploited in Wireless Sensor Networks (WSN), which are traditionally assumed to employ a fixed spectrum allocation and characterized by the communication and processing resource constrains of low-end sensor nodes [3]. Depending on the applications, WSN composed of sensor nodes equipped with CR may benefit from its potential advantages, such as dynamic spectrum access and adaptability for reducing power consumptions. A CR network is formed by $\mathrm{CR}$ nodes that are geographically distributed in a certain area, which is shown in Figure 1. Those nodes are possibly wireless terminals, subscriber users, or sensors in the CR network. In this paper, we propose to employ Distributed Beamforming at CR networks in order to form beams towards Distant CR users so that the CR network is able to forward the signals to DCR users cooperatively. Via adopting the DB method, the CR networks increase its coverage range without causing harmful interferences to PUs.

DB is also referred to as collaborative beamforming, and is originally employed as an energy-efficient scheme to solve long distance transmission in WSN, in order to reduce the amount of required energy and consequently to extend the utilization time of the sensors [4]. The basic idea of DB is that a set of nodes in the wireless network acts as a virtual antenna array and then forms a beam towards a certain direction to collaboratively transmit a signal. DB has been proposed in [4] and it has been shown that by employing $K$ collaborative nodes, the collaborative beamforming can result in up to $K$-fold gain in the received power at a distant access point. Recently a crosslayer approach for DB in wireless ad-hoc networks has been discussed in [5] applying two communication steps. In the first phase, nodes transmit locally in a random access time-slotted fashion. In the second phase, a set of collaborating nodes, acting as a distributed antenna system, forward the received signal to one or more far away destinations. The improved beam pattern and connectivity properties have been shown in [6], and a reasonable

beamforming performance affected by nodes synchronization errors has been discussed in [7]. DB has also been introduced in relay communication systems. 


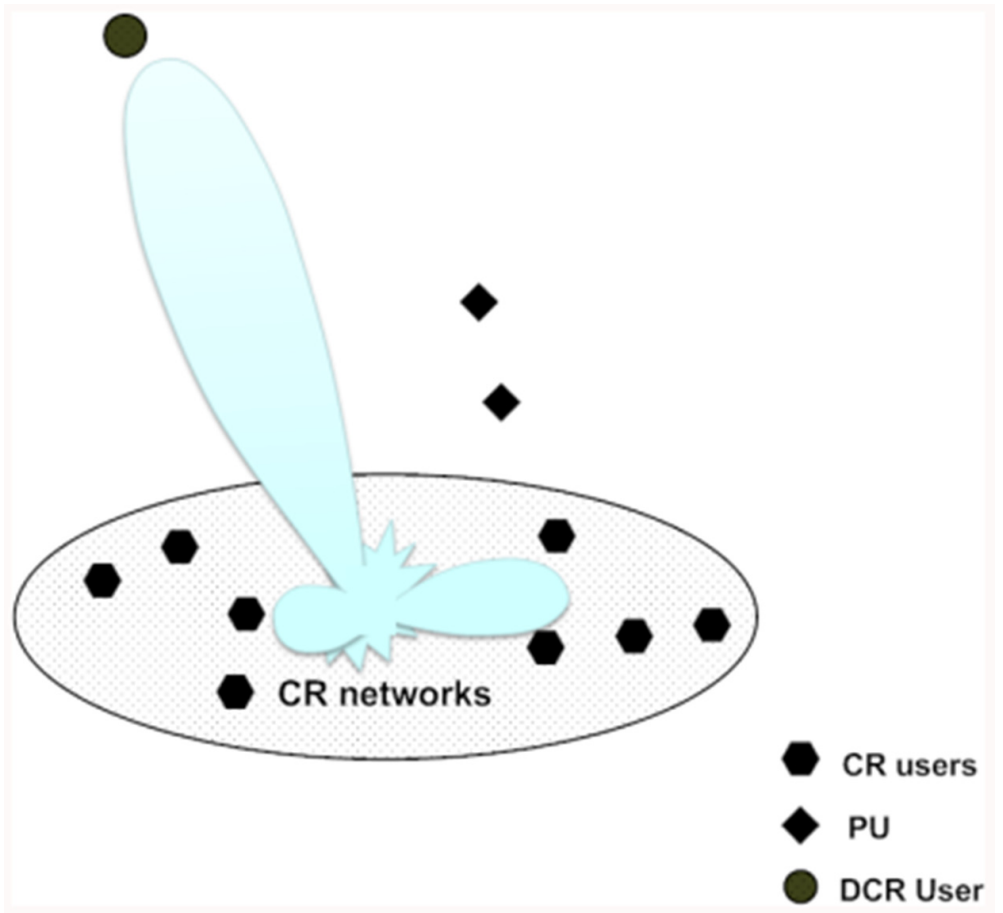

Figure 1 Coexistence of PUs and CR with DB techniques

Different types of relays have been considered, e.g. Amplify-and-Forward (AF) relays, Filter and Forward (FF) relays, etc. The models discussing relay networks in [8-12] have been proposed to have a source, a relay, and a destination, where transmit DB is employed both at the source and at the relay. The authors in [8-11] have developed several DB techniques for relay networks with flat fading channels. In [12], frequency selective fading has been considered.

DB requires accurate synchronization; in other words, the nodes must start transmitting at the same time, synchronize their carrier frequencies, and control their carrier phases so that their signals can be combined constructively at the destination. A synchronization technique based on time-slotted roundtrip carrier synchronization has been proposed for DB in[13], and a review has been given in [14]. In this paper, we adopt the master-slave architecture proposed in [7], where a designated master transmitter (one of CR nodes in the networks) coordinates the synchronization of others (slave) transmitters for DB. This method has also been proved in [7] that a large fraction of DB 
gains can still be realized even with imperfect synchronization corresponding to phase errors with moderately large variance.

In [4], the authors demonstrate that the sidelobe level in the beampattern generated by the DB method will approach $\frac{1}{K}$. where $K$ is the number of cooperating nodes. Thus as long as PUs are not located in the same direction as the beampattern for the DCR user, the transmit power arriving at PUs' directions will always be $K-1$ times less than that towards the DCR users. As a result, it is not necessary to direct specific nulls towards directions of PUs; the DB method by its nature is capable of guaranteeing $K-1$ times less power towards PUs compared with that towards DCR users. However, to apply the DB technique in CR networks, a practical difficulty rather than nulls directing rises due to the fact that the width of the main beam in the beampattern generated by the DB method is relying on the working frequency of the CR networks. As also shown in [4], the main beam of the beampattern will become narrower when $\widetilde{R}$ increases, where $\widetilde{R} \triangleq R / \lambda, R$ is the covering radius of the network and $\lambda$ is the wavelength. If we consider a CR network with $R=100 \mathrm{~m}$ and utilizing the spectrum of the Ultra High Frequency (UHF) band, which is, for instance, $750 \mathrm{MHz}$, we can obtain that $\widetilde{R}=250$. In [4], the authors have shown that the width of the main beam can be approximated by $\frac{35^{\circ}}{\widetilde{R}}$. Thus in our example, the width of the main beam will become about $0.1^{\circ}$. Such a narrow main beam implies that once the DOA estimation of the distant nodes is not accurate enough, the main beam in the pattern may miss its direction. Meanwhile, it also reveals that the width of the main beam in the beampattern mostly relies on the center frequency at which the CR network is able to access.

To solve this problem of the extreme narrow main beam, we propose a novel Nodes Selection (NS) method. The presented NS method is based on the differences in beam width of a broadside array and an end-fire array. The beampatterns of these two types of arrays allow us to find out how the main beam of the end-fire array is much wider than that of the broadside array. We thus conclude that the "broadside" size of the CR network should be small so that a beampattern with a wider main beam can be maintained. As a result, we suggest selecting CR nodes, which are able to form a full size end-fire array and a reduced size broadside array. In other words, we choose those CR nodes which are located in the "belt" area along the direction of the distant nodes.

The paper is arranged as follows. Section 2 introduces DB technique to CR networks. A novel NS method to enlarge the main beam of the beampattern generated by the DB technique is presented in section 3. Simulations will be given afterwards in section 4, showing that our NS method is effective in 
generating a wider main beam in the beam pattern. Section 5 concludes the whole chapter.

\section{DB for CR Networks}

The geometrical structure of the first model together with distant receiver terminals including PUs and DCR users is illustrated in Figure 2. $K$ CR nodes are uniformly distributed over a disc centered at $\mathrm{O}$ with radius $R$. We denote the polar coordinates of the $k$ th CR node by $\left(r_{k}, \Psi_{k}\right)$. The number of DCR users is $L_{D C R}$. These DCR users are considered as access points, and located in the same plane at $\left(A_{i}^{D C R}, \phi_{i}^{D C R}\right), i=1,2, \ldots, L_{D C R}$. Meanwhile the number of PUs is $L_{P U}$, and these users coexist with DCR users. Their locations are $\left(A_{i}, \phi_{i}\right), i=1,2, \ldots, L_{P U}$. The $\mathrm{CR}$ nodes in the $\mathrm{CR}$ network are requested to form a virtual antenna array and collaboratively transmit a common message $s(t)$.

\subsection{Necessary assumptions}

Without loss of generality, we adopt the following assumptions:

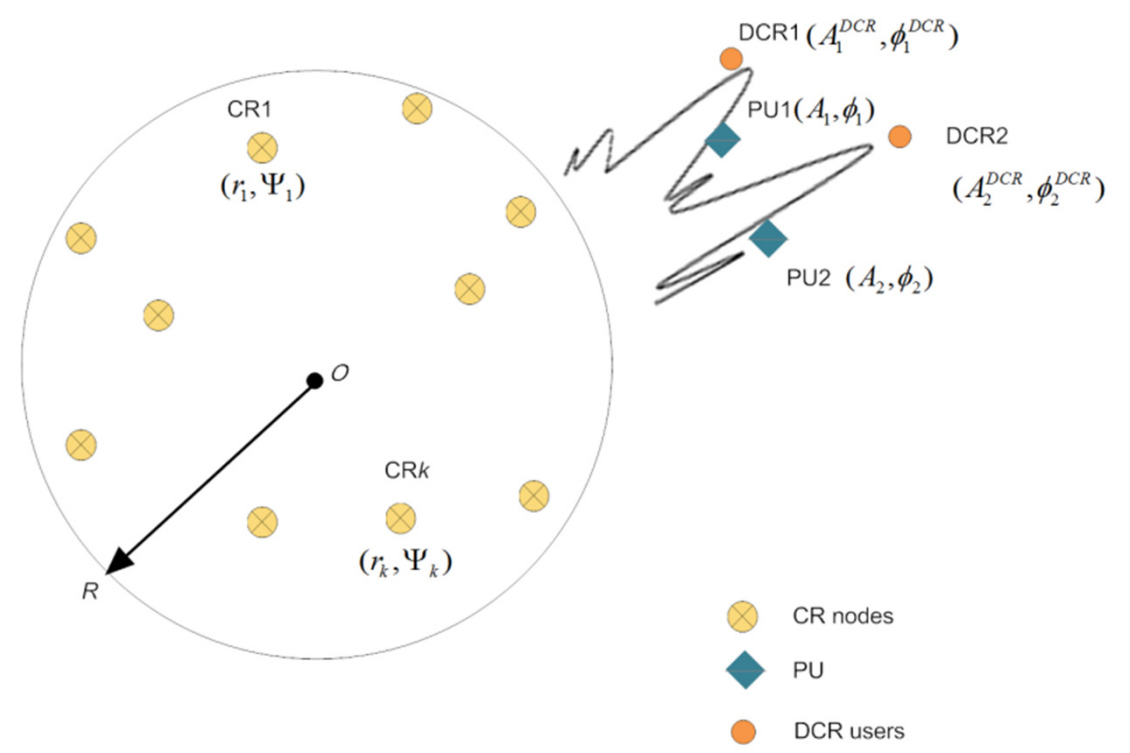

Figure 2 CR networks with DCR users and PUs 
1) The number of $C R$ nodes are larger than that of DCR users, i.e., $K>L_{D C R}$. This is required to solve the multi beam generating problem which has been discussed in [15].

2) All DCR users and PUs are located in the farfield of the CR network, such that $A_{i}^{D C R}>>R, i=1,2, \ldots, L_{D C R}$ and $A_{i}>>R, i=1,2, \ldots, L_{P U}$

3) The bandwidth of $s(t)$ is narrow, so that $s(t)$ is considered to be constant during the time interval $R / c$, where $c$ is the speed of light. However, it has been discussed in [16] that the OFDM scheme is the first recommended candidate for use in the CR network. Since the OFDM signal can be regarded as a combination of narrow band modulated signals, the proposed method in this paper can also be implemented in wide band $\mathrm{CR}$ OFDM systems.

\subsection{DB for CR networks}

Let $x_{k}(t)$ denote the transmitted signal from the $k$ th node,

$$
x_{k}(t)=s(t) e^{j 2 \pi f t}
$$

where $f$ is the carrier frequency. The received signal at an arbitrary point $(A, \phi)$ in the far field due to the $k$ th node transmission is [17]

$$
r_{k}(t)=\beta_{k} x_{k}\left(t-\frac{d_{k}}{c}\right)=\beta_{k} s\left(t-\frac{d_{k}}{c}\right) e^{j 2 \pi f t} e^{-j \frac{2 \pi}{\lambda} d_{k}}
$$

where $d_{k}$ is the distance between the $k$ th node and the access point $(A, \phi)$, and $\beta_{k}=\left(d_{k}\right)^{\frac{-\gamma}{2}}$ is the signal path loss with $\gamma$ donating the path loss exponent. Making use of assumption 2 in the previous paragraph [17], $\beta_{k}$ and $d_{k}$ are approximated by

$$
\begin{gathered}
d_{k}=\sqrt{A^{2}+r_{k}^{2}-2 A r_{k} \cos \left(\phi-\Psi_{k}\right)} \approx A-r_{k} \cos \left(\phi-\Psi_{k}\right) \\
\beta_{k}=\left(d_{k}\right)^{\frac{-\gamma}{2}} \approx\left[A-r_{k} \cos \left(\phi-\Psi_{k}\right)\right]^{\frac{-\gamma}{2}} \approx \beta\left(1+\frac{\gamma r_{k} \cos \left(\phi-\Psi_{k}\right)}{2 A}\right)
\end{gathered}
$$

where $\beta=A^{\frac{-\gamma}{2}}$. It is also ensured that $\frac{\gamma r_{k} \cos \left(\phi-\Psi_{k}\right)}{2 A}<<1$. Thus $\beta_{k}$ can then be approximated by $\beta$. Substituting equation (3) and (4) into equation (2), it follows that [17],

$$
r_{k}(t) \approx \beta e^{-j \frac{2 \pi}{\lambda} A} s\left(t-\frac{A}{c}\right) e^{j 2 \pi f t} e^{j \frac{2 \pi}{\lambda} r_{k} \cos \left(\phi-\Psi_{k}\right)}
$$


We assume there is only one DCR, i.e., $L_{D C R}=1$, and simplify $\left(A_{1}^{D C R}, \phi_{1}^{D C R}\right)$ by $\left(A_{0}, \phi_{0}\right)$. The case with more than one DCR users has been discussed in [15]. As proposed in [4], we adopt for the initial phase of each node

$$
\varphi_{k}=-\frac{2 \pi}{\lambda} r_{k} \cos \left(\phi_{0}-\Psi_{k}\right)
$$

The received signal $r_{k}(t)$ at $(A, \phi)$ becomes

$$
r_{k}(t) \approx \beta e^{-j \frac{2 \pi}{\lambda} A} s\left(t-\frac{A}{c}\right) e^{j 2 \pi f t} e^{j \frac{2 \pi}{\lambda} r_{k} \cos \left(\phi-\Psi_{k}\right)} e^{-j \frac{2 \pi}{\lambda} r_{k} \cos \left(\phi_{0}-\Psi_{k}\right)}
$$

The array factor $F\left(\phi \mid r_{k}, \Psi_{k}\right)$ yields:

$$
\begin{aligned}
F\left(\phi \mid r_{k}, \Psi_{k}\right) & \approx \frac{1}{K} \sum_{k=1}^{K} e^{j \frac{2 \pi}{\lambda} r_{k}\left[\cos \left(\phi-\Psi_{k}\right)-\cos \left(\phi_{0}-\Psi_{k}\right)\right]} \\
& =\frac{1}{K} \sum_{k=1}^{K} e^{-j \frac{4 \pi}{\lambda} r_{k} \sin \left(\frac{\phi-\phi_{0}}{2}\right) \sin \left(\frac{\phi+\phi_{0}-2 \Psi_{k}}{2}\right)}
\end{aligned}
$$

We assume there are many CR nodes and the locations of CR nodes follow a uniform distribution over a disk of radius $R$, leading to the probability density functions (pdf)

$$
\left\{\begin{array}{c}
f_{r_{k}}(r)=\frac{2 r}{R^{2}}, 0 \leq r<R \\
f_{\Psi_{k}}\left(\Psi_{k}\right)=\frac{1}{2 \pi}, \quad-\pi \leq \Psi_{k}<\pi
\end{array}\right.
$$

By defining $z_{k} \triangleq \frac{r_{k}}{R} \sin \left(\Psi_{k}-\frac{\phi_{1}+\phi_{0}}{2}\right)$, the compound random variable $z_{k}$ has a pdf [4]

$$
f_{z_{k}}\left(z_{k}\right)=\frac{2}{\pi} \sqrt{1-z_{k}^{2}}, \quad-1 \leq z<1
$$

The array factor in equation (8) can now be written as

$$
F\left(\phi \mid z_{k}\right)=\frac{1}{K} \sum_{k=1}^{K} \exp \left(-j 4 \pi \widetilde{R} \sin \left(\frac{\phi-\phi_{0}}{2}\right) z_{k}\right)
$$

where $\widetilde{R} \triangleq R / \lambda$ is the radius of the disk normalized by the wavelength. The far field beampattern is defined by

$$
P\left(\phi \mid z_{k}\right) \triangleq\left|F\left(\phi \mid z_{k}\right)\right|^{2}
$$


and the average array beam pattern of the CR networks becomes [4]

$$
P_{a v}(\phi) \triangleq \mathrm{E}\left[P\left(\phi \mid z_{k}\right)\right]=\frac{1}{K}+\left(1-\frac{1}{K}\right) \mu^{2}(\phi)
$$

where

$$
\begin{gathered}
\mu(\phi)=E[F(\phi)]=\left|\frac{2 J_{1}(\alpha(\phi))}{\alpha(\phi)}\right| \\
\alpha(\phi) \triangleq 4 \pi \widetilde{R} \sin \left(\frac{\phi-\phi_{0}}{2}\right)
\end{gathered}
$$

$J_{n}(\cdot)$ stands for the $n$th order Bessel function of the first kind, and $E[\cdot]$ stands for the statistical expectation. Above equations learn us that if each CR node adopts the initial phase as given in equation (6), the average pattern generated by the whole CR network can be obtained from equation (13).

Figure 3 shows the average beampattern of the DB method for different $K(K=8,16)$ and $\widetilde{R}(\widetilde{R}=2,4,8)$. We assume that the direction of DCR is $\phi_{0}=0^{\circ}$. It can be seen that when the beam angle moves away from the

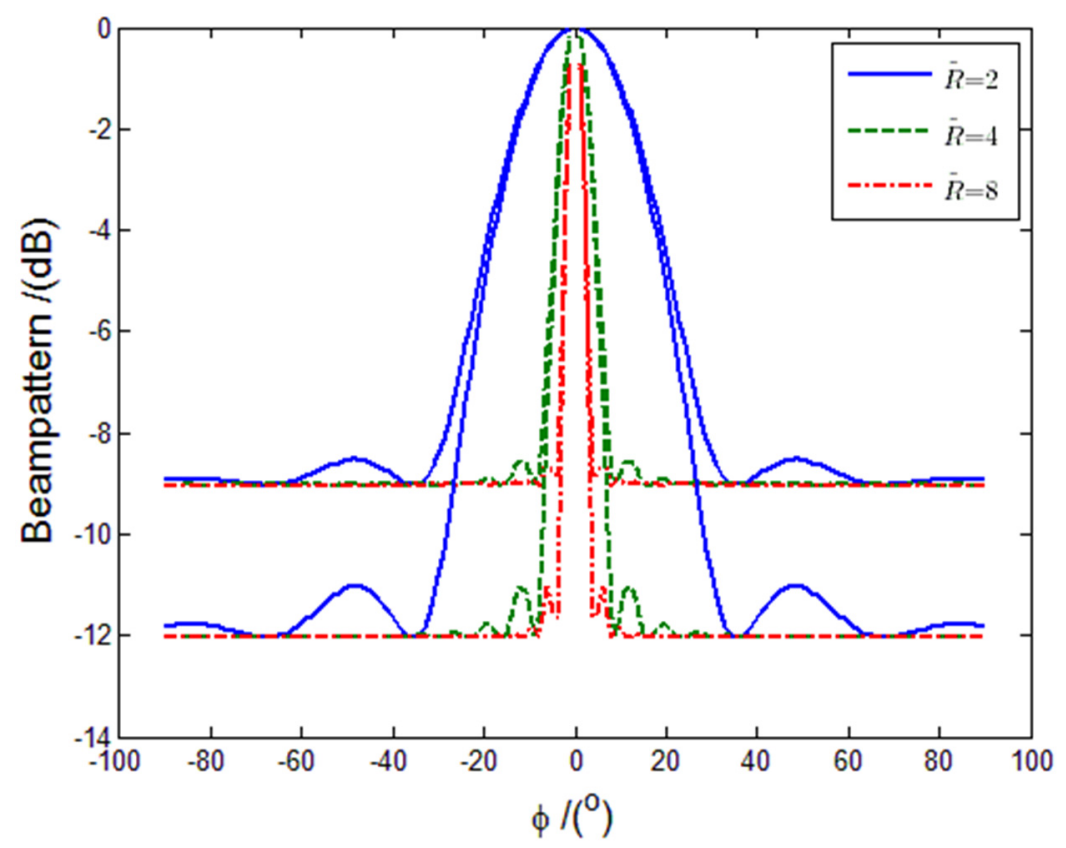

Figure 3 Average beampattern of the DB method 
direction of DCR, the sidelobe approaches $\frac{1}{K}$, i.e., $10 \log _{10}\left(\frac{1}{8}\right) \approx-9 d B$ and $10 \log _{10}\left(\frac{1}{16}\right) \approx-12 d B$, respectively. This leads to the logical conclusions that the sidelobe level decreases when $K$ increases, and that the larger $\widetilde{R}$ becomes, the narrower the main beam will be, and consequently the better directivity in the beampattern will be achieved. Figure 3 is only for demonstration purposes, because the value of the parameters that we considered ( $K=8,16 ; \widetilde{R}=2,4,8)$ are not realistic in the real application. In practical applications, $\widetilde{R}$ should be large enough so that CR networks can have enough CR nodes.

\section{NS for CR Networks with Enlarged Main Beam}

In this section we propose a new NS method for CR networks to select proper nodes to achieve wider main beam in order to solve the problem of $\widetilde{R}$ increasing rapidly considering the higher operating frequency of the CR networks. We first consider two extreme cases of the CR network, which are two types of array antennas: broadside array and end-fire array by projecting the location of each $\mathrm{CR}$ node along an $\mathrm{X}$ and $\mathrm{Y}$ axis, and study the properties of these two array antennas. Next we propose the NS method.

We assume one DCR user is located along the $\mathrm{X}$ axis $\left(\phi_{0}=0^{\circ}\right)$. We then consider the location of a CR node by projecting it into the Cartesian coordinate system (X and $\mathrm{Y}$ directions) as shown in Figure 4. In this way we create two virtual arrays: broadside array and end-fire array. We now discuss the performance of the two separate arrays (broadside array and end-fire array) instead of the full CR network.

The average beampatterns of these two arrays are summarized in the following equations

$$
\bar{P}_{\text {broadside }}(\phi)=\frac{1}{K}+\left(1-\frac{1}{K}\right) \mu_{b}^{2}(\phi)
$$

where

$$
\begin{gathered}
\mu_{b}(\phi)=\left|\frac{2 J_{1}\left(\alpha_{b}(\phi)\right)}{\alpha_{b}(\phi)}\right| \\
\alpha_{b}(\phi)=2 \pi \widetilde{R}\left(\sin \phi-\sin \phi_{0}\right)
\end{gathered}
$$

and

$$
\bar{P}_{\text {end-fire }}(\phi)=\frac{1}{K}+\left(1-\frac{1}{K}\right) \mu_{e}^{2}(\phi)
$$




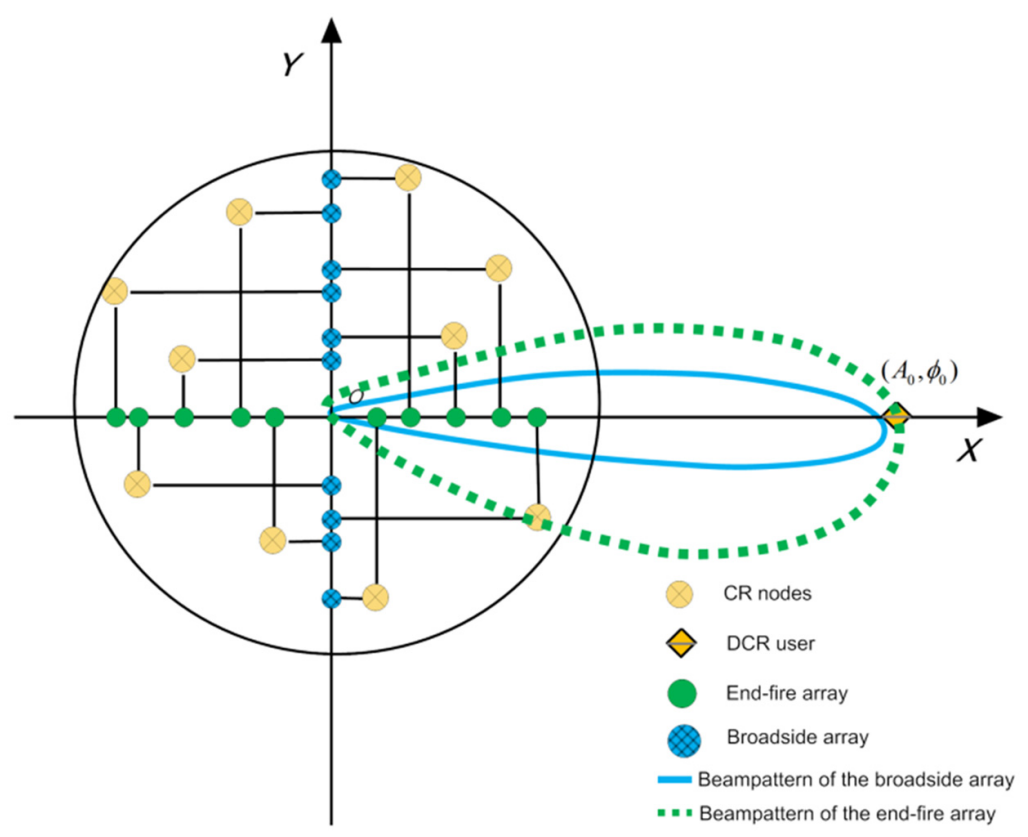

Figure 4 Converting locations of CR nodes into broadside and end-fire arrays

where

$$
\begin{gathered}
\mu_{e}(\phi)=\left|\frac{2 J_{1}\left(\alpha_{e}(\phi)\right)}{\alpha_{e}(\phi)}\right| \\
\alpha_{e}(\phi)=2 \pi \widetilde{R}\left(\cos \phi-\cos \phi_{0}\right)
\end{gathered}
$$

Proofs of equations (16) to (21) can be found in Appendix A.

The results of equation (16) and (21) are shown in Figure 5. We assume there are 32 nodes in the CR network and the normalized radius is 35, i.e., $K=32$ and $\widetilde{R}=35$. We also assume there is only one DCR user, and its DOA is $\phi_{0}=0^{\circ}$. We can conclude from Figure 5 that the broadside array has the same average beampattern as the previous CR network. This is due to the result shown in equation (16). The $\alpha_{b}(\phi)$ in equation (18) can be approximated to $\alpha(\phi)$ defined in equation (15), when $\phi$ is close to $\phi_{0}$. As a result in the angle area close to $\phi_{0}=0^{\circ}$, the performances of the beampattern of the broadside array and the DB method are very similar to each other. In addition, to the same conclusion that has also been drawn in [18], we have discovered that the width of the main beam is in a reverse relationship with the size of the broadside array. Thus we are inspired by these two facts that if 


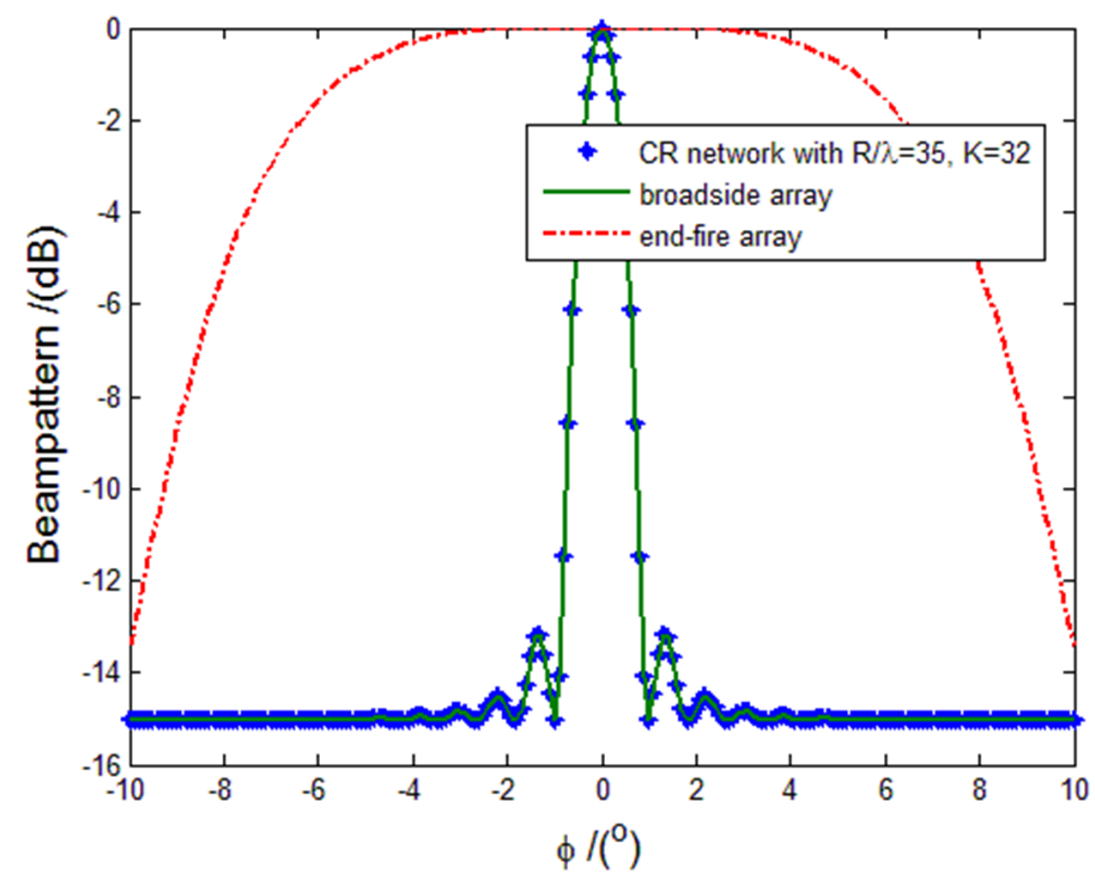

Figure 5 Beampattern of CR network, broadside array and end-fire array

we want to enlarge the width of the main beam, we have to decrease the length (size) of the broadside array and we can adopt the end-fire array instead.

Based on this idea, we propose a NS method and select those nodes, which are able to form a full size end-fire array and a reduced size broadside array. Thus we choose the nodes in a relatively narrower belt along the Direction of Arrival (DOA) of the DCR user, as shown in Figure 6. In Figure 6, the CR nodes are selected in a way that the size of the "broadside" is limited to $D$, where $\frac{D}{\lambda}<\widetilde{R}$. When we consider the case with more than one DCR user coexisting with the CR network, e.g. two DCR users, the NS method is demonstrated in Figure 7. We choose those CR nodes which are in the two "belt" areas as shown in Figure 7. In addition, for those double selected CR nodes, which are in the cross area, we adopt the method which let them randomly choose one of the two DCR users to serve, which has been proposed and discussed in [15].

When we consider more than two DCR users, the NS method is slightly different than the case of two DCR users. Figure 8 demonstrates the idea of how to select CR nodes utilizing our proposed NS method, when there are three present DCR users. As we can see from Figure 8, there are three different 


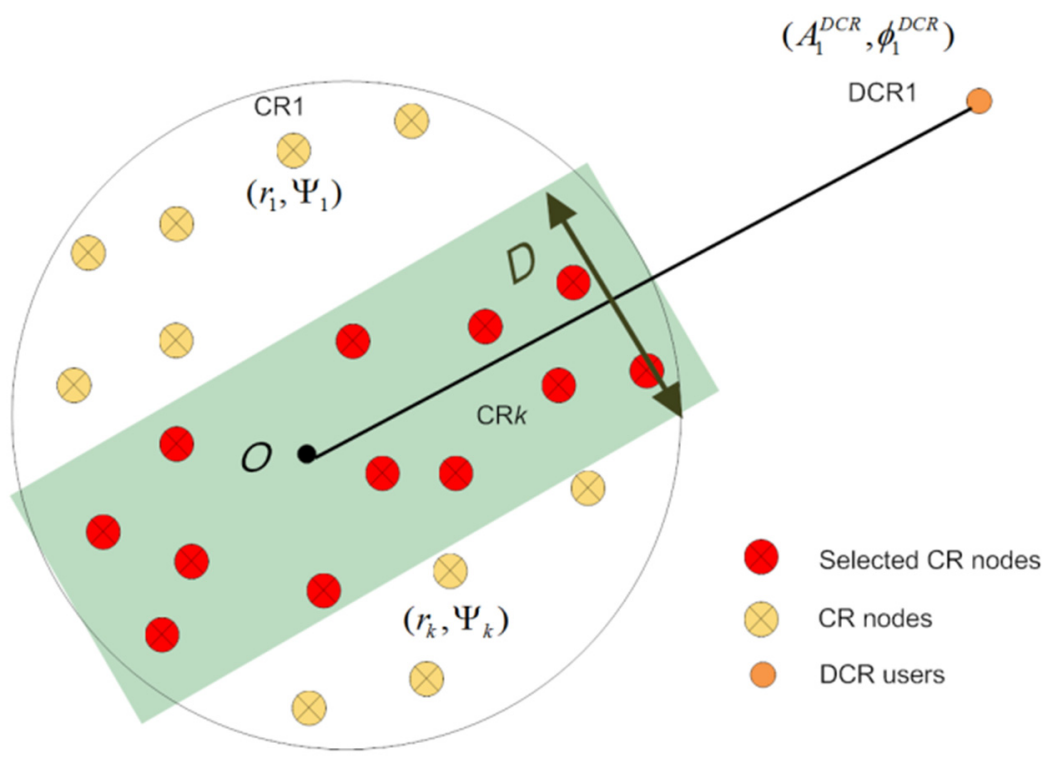

Figure 6 NS for CR networks

types of CR nodes in the CR network. They are nodes which are only selected once in the belt area along one DCR user, and are requested to generate main beams towards this DCR user. They are also nodes which are double selected and triple selected. For the double selected nodes, they can choose between two DCR users, and we let them randomly choose towards which DCR user they want to direct their main beam in the pattern. For the triple selected CR nodes, they have three options of different DCR users to choose and again we let them choose among these three. It is worth noticing that double and triple selected nodes have different sets of DCR users to choose from.

\section{Simulation Results of the NS Method}

Figure 9 and 10 demonstrate the selected nodes in the CR networks with different values of $D$ by adopting the proposed NS method. Considering the fact that the number of selected nodes varies from each simulation, we do averaging in order to show a general result of the beampattern. As a result, the beampattern of the NS method shown in Figure 11 is the average beampattern. In Figures 9-10, we assume there is only one DCR user and its DOA is $\phi_{0}=0^{\circ}$. We choose the nodes within the width of the belt $D=15 \lambda$ and $D=35 \lambda$, 


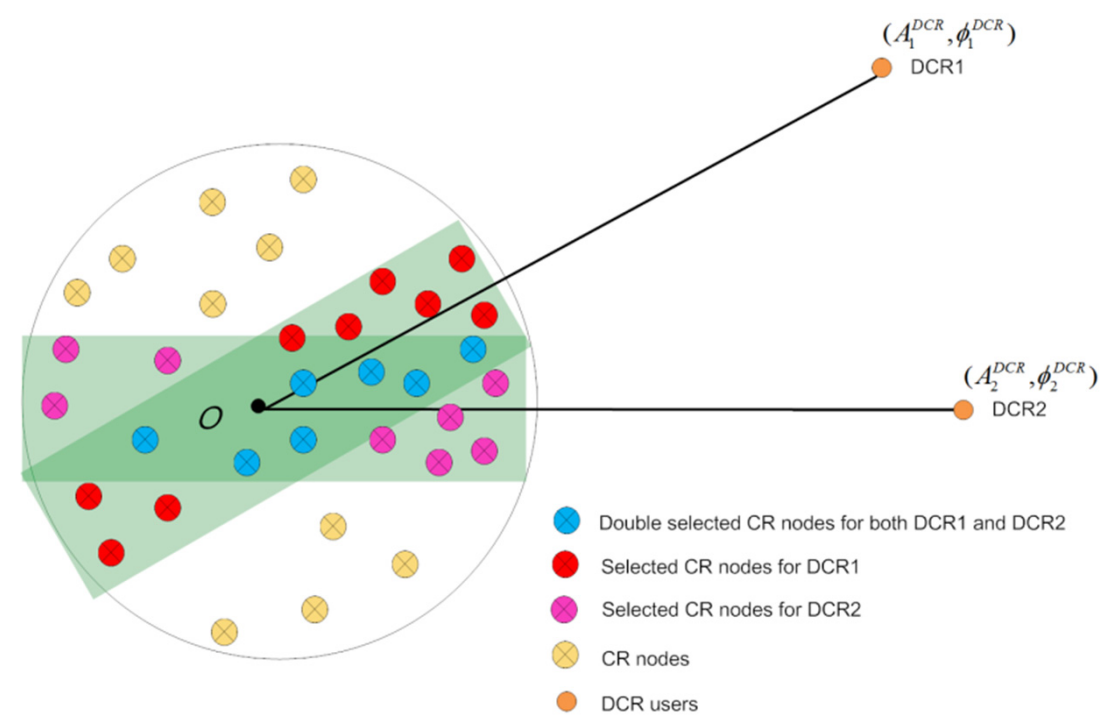

Figure 7 NS for CR networks with two DCR users

as shown in Figure 9 and 10, respectively. We assume there are 60 nodes in the network and the normalized radius of the network is 50, i.e., $K=60$, and $\tilde{R}=50$. The result of the beampattern shown in Figure 11 is the average of 1000 runs.

Figure 9and 10 show that those CR nodes, which are located in the belt area, as defined in Figure 6, are successfully selected for transmission. We can also see from these two figures that when $D$ is smaller, less number of CR nodes will be selected for transmission.

Figure 11 shows that after adopting the proposed NS method, the main beam in the beampattern of the DB method is enlarged. Employing the NS method with defined $D=10 \lambda, D=15 \lambda$ and $D=35 \lambda$, the main beam is about six times, four times and two times wider than that without adopting the NS method, respectively. However, with smaller $D$, less CR nodes will be selected, as shown in Figure 6. Therefore, the beampattern has a higher sidelobe level than that with a larger $D$, since the asymptotic sidelobe level of the beampattern is proportional to the reverse of the number of nodes of the CR network, as explained in Figure 3. With smaller $D$, which selects fewer CR nodes for transmission, the width of the main beam is the largest. This shows that NS is a trade-off between the width of the main beam and the sidelobe level in the beampattern. 


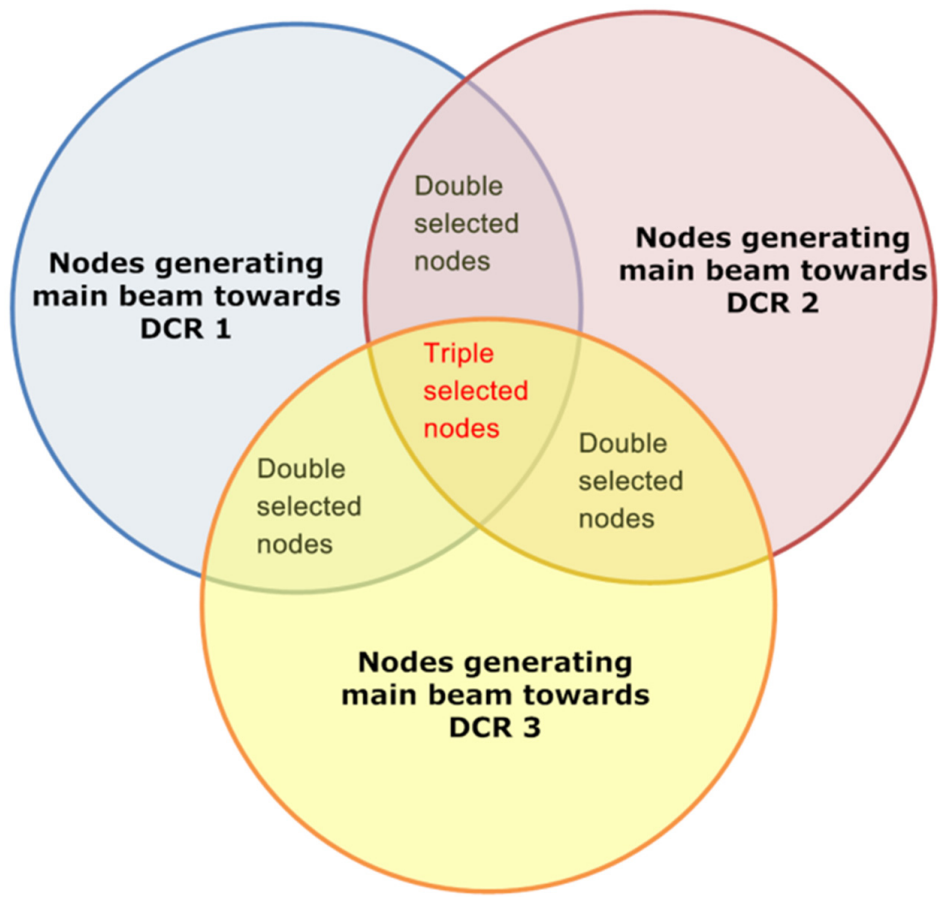

Figure 8 Demonstration of NS method with three DCR users

Figure 12 and 13 consider the case with two DCR users, which are located at $\phi_{1}=0^{\circ}$ and $\phi_{2}=15^{\circ}$ when $D=15 \lambda$. Figure 12 shows the selected nodes in the CR networks to participate in CR transmission towards two DCR users. We can see in Figure 12 that there are a few CR nodes which are double selected for participating in transmission towards both DCR users.

Figure 13 shows the average beampattern of the NS method for two DCR users. As can be seen fromthis figure, the two main beams in the beampattern are directed towards $\phi_{1}=0^{\circ}$ and $\phi_{2}=15^{\circ}$, respectively. The main beams are both widened via adopting our NS method. When we employ NS method with $D=15 \lambda$, we can see from Figure 13 that both two main beam are broadened to $3^{\circ}$.

Figure 14 and 15 consider the case with three DCR users, which are located at $\phi_{1}=0^{\circ}, \phi_{2}=15^{\circ}$ and $\phi_{3}=-20^{\circ}$ when $D=15 \lambda$, and show the selected nodes in the CR networks to participate in CR transmission towards these three DCR users. It shows three types of CR nodes as demonstrated in Figure 8.Figure 15 shows the average beampattern of the NS method for three 


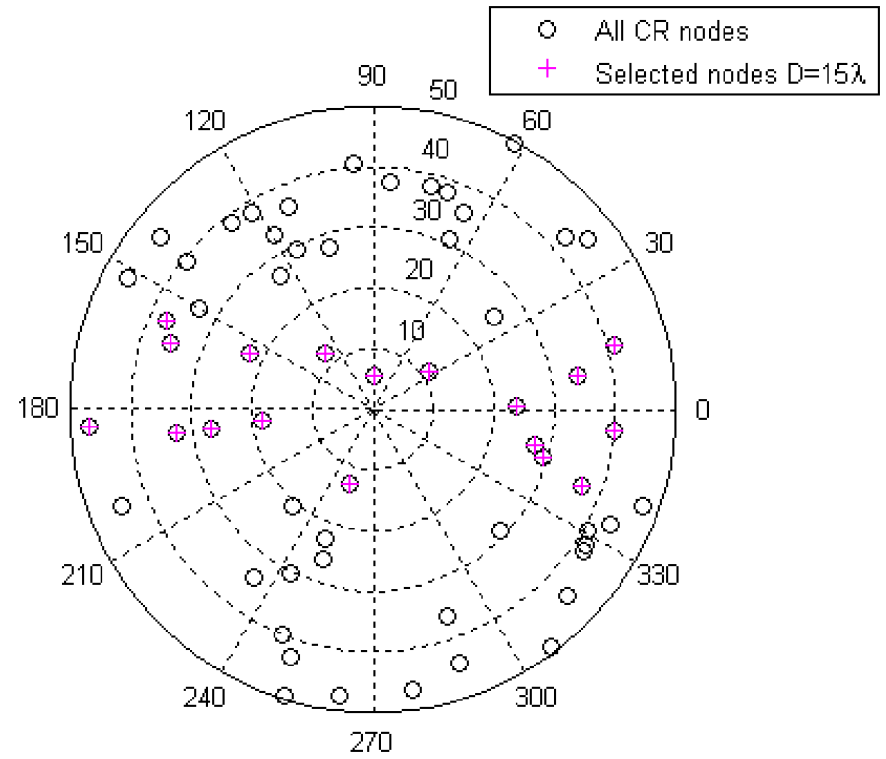

Figure 9 Selected CR nodes in the CR networks $D=15 \lambda\left(\phi_{0}=0^{\circ}\right)$

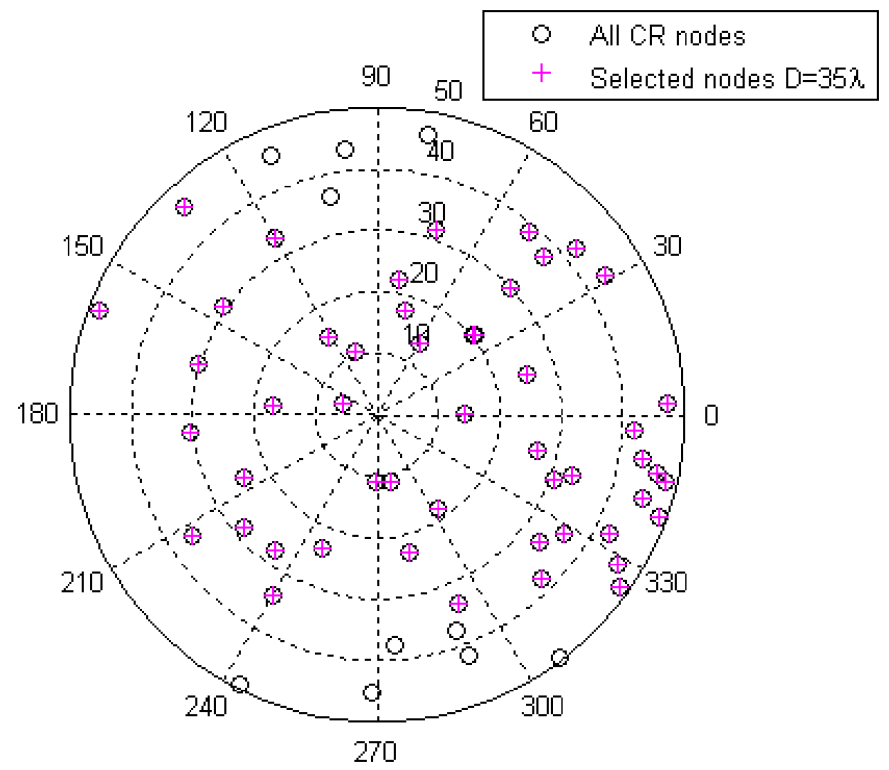

Figure 10 Selected CR nodes in the CR networks $D=35 \lambda\left(\phi_{0}=0^{\circ}\right)$ 


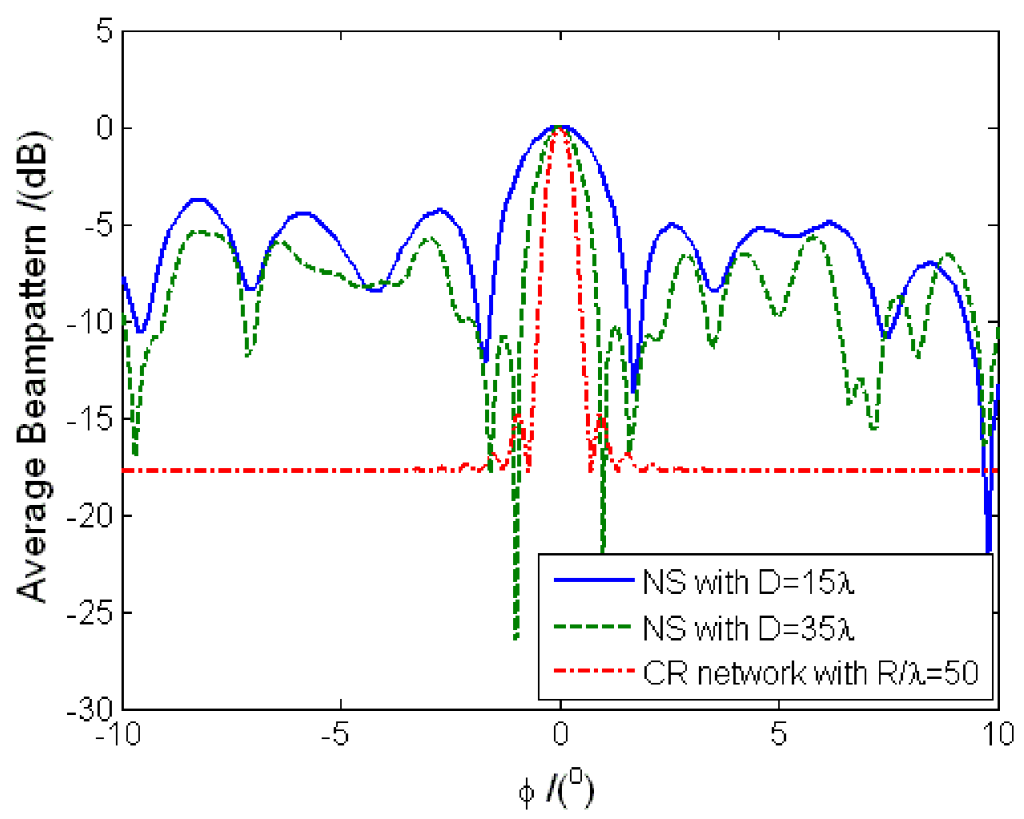

Figure 11 Average beampattern of the selected CR nodes $\left(\phi_{0}=0^{\circ}\right)$

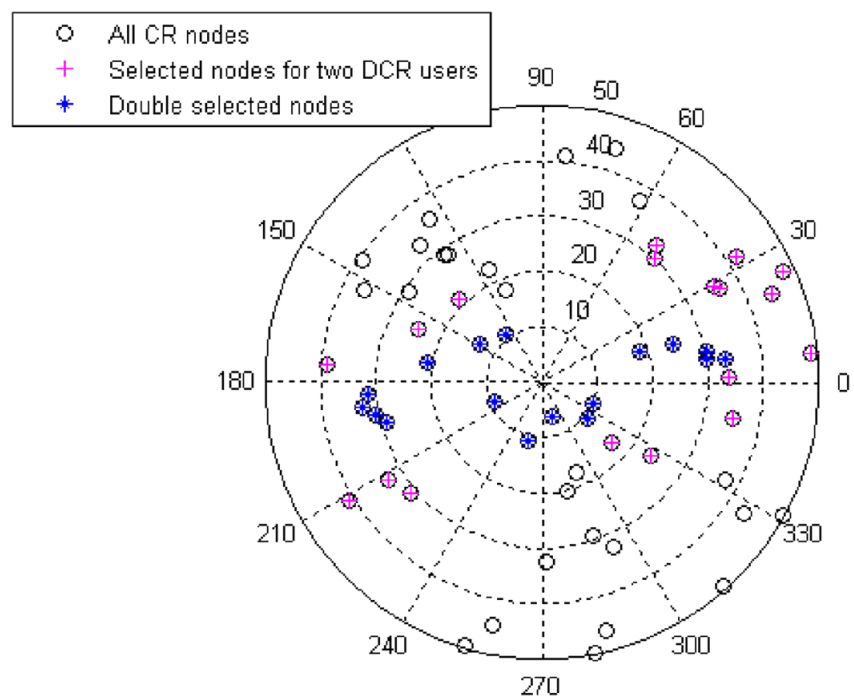

Figure 12 Selected CR nodes in the CR networks $D=15 \lambda\left(\phi_{1}=0^{\circ}\right.$ and $\left.\phi_{2}=15^{\circ}\right)$ 


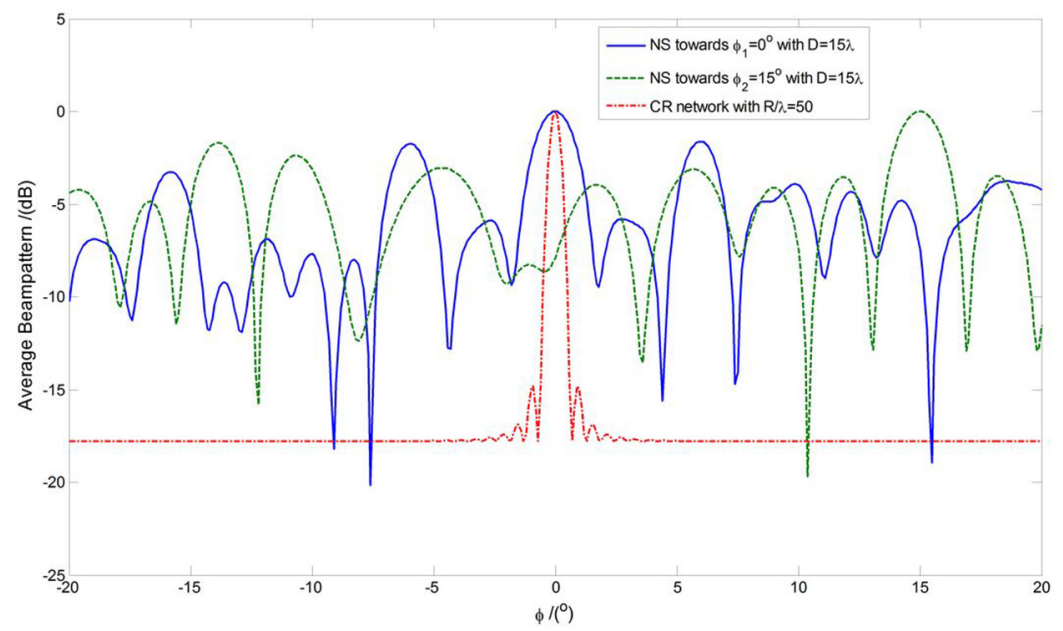

Figure 13 Average beampattern of the selected CR nodes for two DCR users

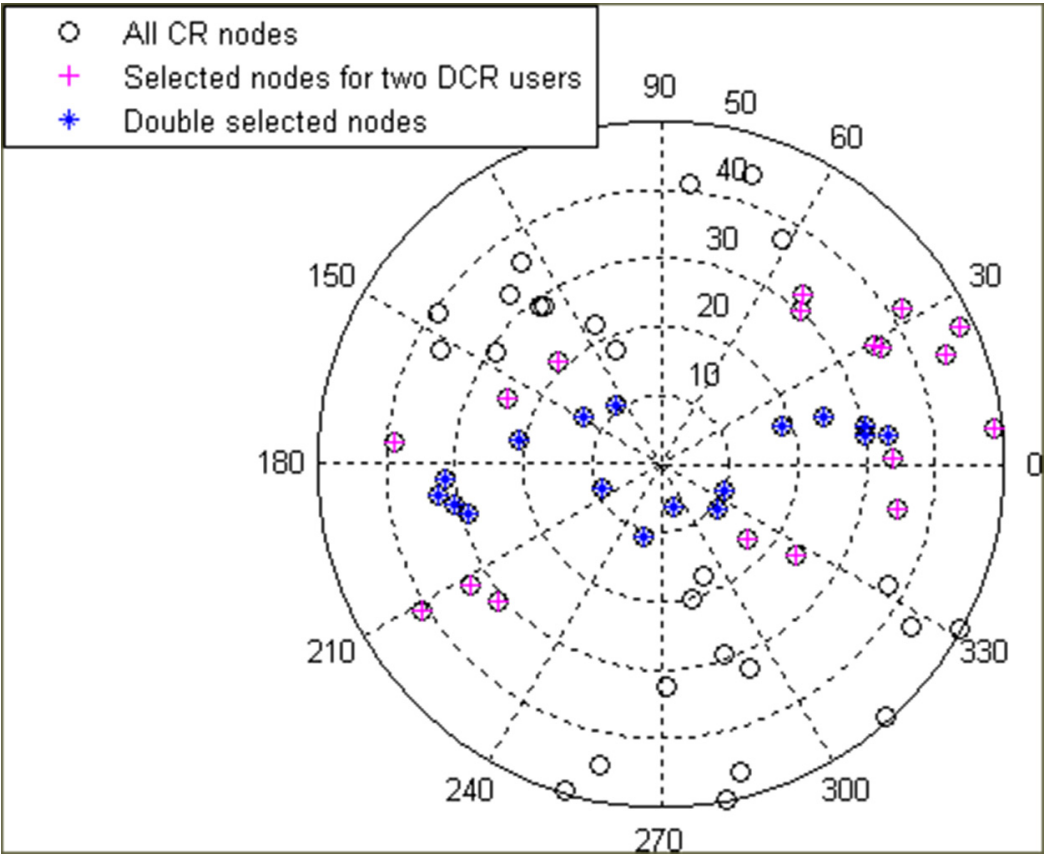

Figure 14 Selected CR nodes in the CR networks $D=15 \lambda\left(\phi_{1}=0^{\circ}, \phi_{2}=15^{\circ}\right.$ and $\phi_{3}=-20^{\circ}$ ) 


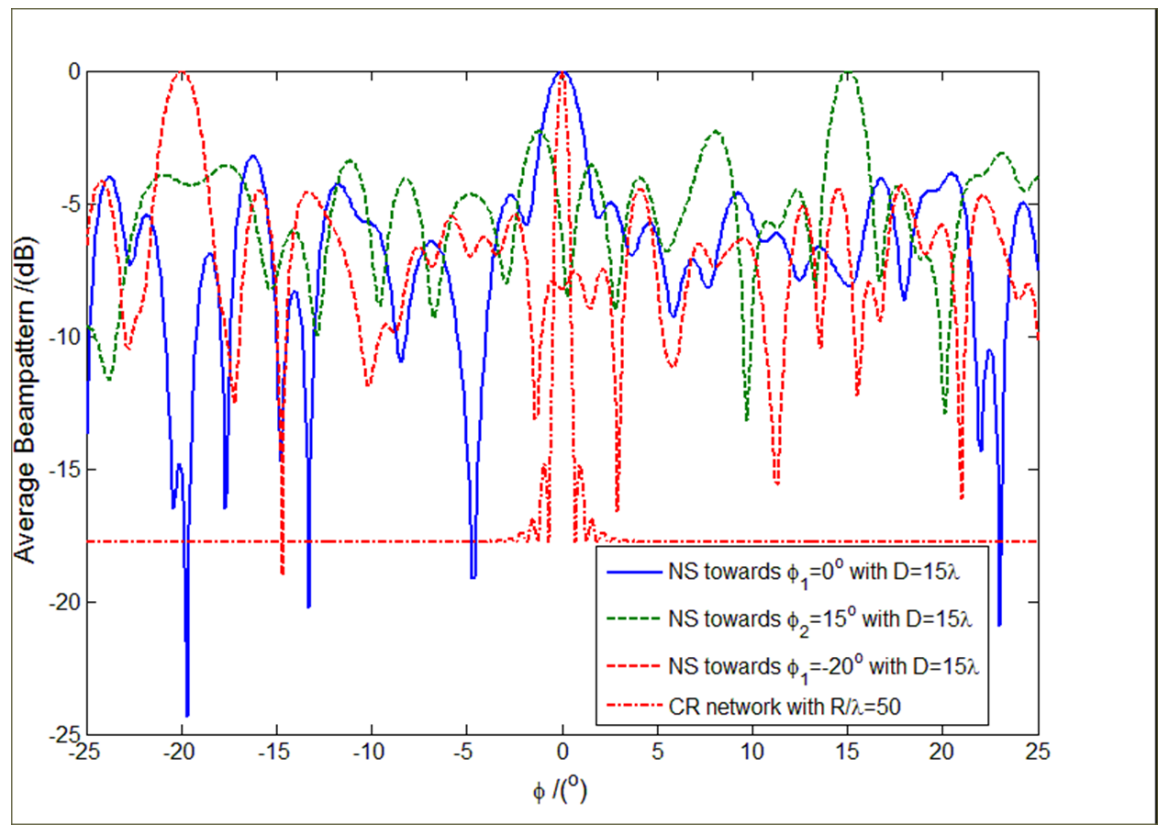

Figure 15 Average beampattern of the selected CR nodes for two DCR users

DCR users. It shows that three main beams in the beampattern are directed towards $\phi_{1}=0^{\circ}, \phi_{2}=15^{\circ}$ and $\phi_{3}=-20^{\circ}$, respectively. All three main beams which are displayed in the pattern towards DCR users are all widened.

Figure 16 shows the result of the average beampattern of our proposed NS method when applied to large CR networks $(\widetilde{R}=100,200,400)$. We consider that the distribution density of the CR nodes remains the same with that of the Figure $9-10(K=60, \widetilde{R}=50)$, and only the size of the networks are increased. Consequently the number of CR nodes of the considered CR networks in Figure 9 and 10 is $K=\frac{60 \times 100^{2}}{50^{2}}=240, K=960$ and $K=3840$. We adopt $D=15 \lambda$ for all the three CR networks. It can be seen from this Figure that the main beams are much wider than those of the DB method without NS method which are approximated by $\frac{35^{\circ}}{\widetilde{R}}$. When the size of the CR network is enlarged, more nodes will be selected to participate in CR transmission. As a result sufficiently lower sidelobes can be achieved. For the case of $\widetilde{R}=400$, far sidelobe levels become approximately $-15 d B$, where near sidelobes are higher. 


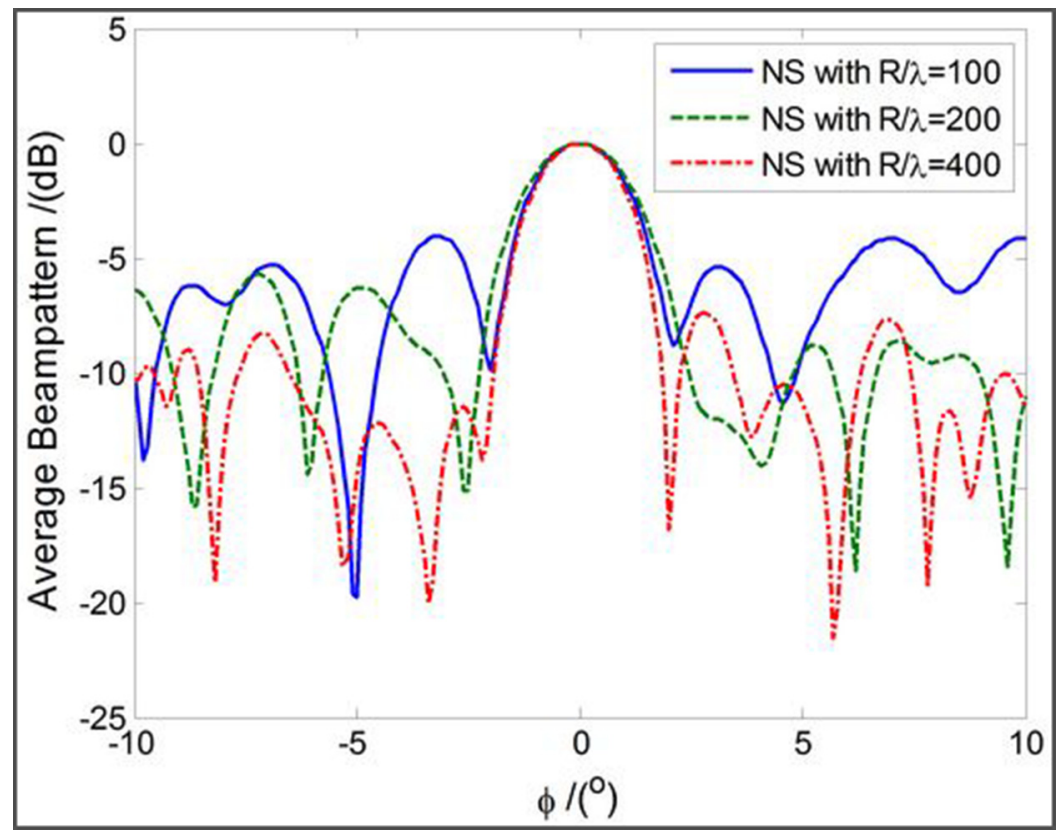

Figure 16 Average beampattern of the selected CR nodes with $\widetilde{R}=100,200,400$

\section{Conclusions}

We have introduced the DB technique to the CR network, which is constituted of distributed CR nodes. The goal of the DB method is to forward the CR signal to the DCR user, while causing no harmful interferences to coexisting PUs by limiting its transmission power towards directions of PUs.

It is unavoidable that introducing the DB method to CR networks will lead to $\tilde{R}$ an extremely narrow main beam of the beampattern. To find a new network structure which has less impact of the working frequency, we have proposed a NS method. The NS method chooses those CR nodes, which are closely located to an end-fire array considering the direction of the DCR user. Our NS method can also be applied to cases with more than one DR users. The average beampattern of the proposed NS method show that the main beams are successfully directed towards the DCR users and are sufficiently enlarged for practical applications in CR networks. What is more, for a CR network with a large physical size, our NS method can widen the main beam while maintaining adequate low sidelobe levels for CR transmission. 


\section{References}

[1] J. Mitola, III, "Cognitive radio: an integrated agent architecture fro software defined radio," Ph.D., Royal institute of technology Stockholm, Sweden.

[2] S. Srinivasa and S. A. Jafar, "The throughput potential of cognitive radio: a theoretical perspective," in Signals, Systems and Computers, 2006. ACSSC '06. Fortieth Asilomar Conference on, 2006, pp. 221-225.

[3] O. Akan, O. Karli, and O. Ergul, "Cognitive radio sensor networks," Network, IEEE, vol. 23, pp. 34-40, 2009.

[4] H. Ochiai, P. Mitran, H. V. Poor, and V. Tarokh, "Collaborative beamforming for distributed wireless ad hoc sensor networks," Signal Processing, IEEE Transactions on, vol. 53, pp. 4110-4124, 2005.

[5] D. Lun, A. P. Petropulu, and H. V. Poor, "A cross-layer approach to collaborative beamforming for wireless Ad Hoc networks," Signal Processing, IEEE Transactions on, vol. 56, pp. 2981-2993, 2008.

[6] K. Zarifi, S. Affes, and A. Ghrayeb, "Distributed beamforming for wireless sensor networks with random node location," in Acoustics, Speech and Signal Processing, 2009. ICASSP 2009. IEEE International Conference on, 2009, pp. 2261-2264.

[7] R. Mudumbai, G. Barriac, and U. Madhow, "On the feasibility of distributed beamforming in wireless networks," Wireless Communications, IEEE Transactions on, vol. 6, pp. 1754-1763, 2007.

[8] J. Yindi and H. Jafarkhani, "Network beamforming using relays with perfect channel information," Information Theory, IEEE Transactions on, vol. 55, pp. 2499-2517, 2009.

[9] Z. Gan, W. Kai-Kit, A. Paulraj, and B. Ottersten, "Collaborative-relay beamforming with perfect CSI: optimum and distributed implementation," Signal Processing Letters, IEEE, vol. 16, pp. 257-260, 2009.

[10] V. Havary-Nassab, S. Shahbazpanahi, A. Grami, and L. Zhi-Quan, "Distributed beamforming for relay networks based on second-order statistics of the channel state information," Signal Processing, IEEE Transactions on, vol. 56, pp. 4306-4316, 2008.

[11] S. Fazeli-Dehkordy, S. Shahbazpanahi, and S. Gazor, "Multiple peer-to-peer communications using a network of relays," Signal Processing, IEEE Transactions on, vol. 57, pp. 3053-3062, 2009.

[12] C. Haihua, A. B. Gershman, and S. Shahbazpanahi, "Filter-andForward distributed beamforming in relay networks with frequency 
selective fading," Signal Processing, IEEE Transactions on, vol. 58, pp. 1251-1262, 2010.

[13] D. R. Brown and H. V. Poor, "Time-slotted round-trip carrier synchronization for distributed beamforming," Signal Processing, IEEE Transactions on, vol. 56, pp. 5630-5643, 2008.

[14] R. Mudumbai, D. R. Brown, U. Madhow, and H. V. Poor, "Distributed transmit beamforming: challenges and recent progress," Communications Magazine, IEEE, vol. 47, pp. 102-110, 2009.

[15] X. Lian, H. Nikookar, and L. P. Ligthart, "Efficient radio transmission with adaptive and distributed beamforming for Intelligent WiMAX," Wirel. Pers. Commun., vol. 59, pp. 405-431, 2011.

[16] M. Jun, G. Y. Li, and J. Biing Hwang, "Signal processing in cognitive radio," Proceedings of the IEEE, vol. 97, pp. 805-823, 2009.

[17] K. Zarifi, S. Affes, and A. Ghrayeb, "Collaborative null-steering beamforming for uniformly distributed wireless sensor networks," Signal Processing, IEEE Transactions on, vol. 58, pp. 1889-1903, 2010.

[18] C. A. Balanis, Antenna Theory: Analysis and Design, 3rd Edition: WileyInterscience, 2005.

\section{Appendix A}

Proofs of Equations (16)-(21)

We assume the Cartesian Coordinates of the $k$ th CR node to be $\left(r_{k, x}, r_{k, y}\right)$, where

$$
\begin{aligned}
& r_{k, x}=r_{k} \cos \Psi_{k} \\
& r_{k, y}=r_{k} \sin \Psi_{k}
\end{aligned}
$$

Using equation (6), we can further separate the initial phase of the $k$ th CR node into two parts, which are dedicated to the broadside array $\varphi_{k, b}$ and to the end-fire array $\varphi_{k, e}$. It means

$$
\varphi_{k}=\varphi_{k, b}+\varphi_{k, e}
$$

where

$$
\begin{aligned}
\varphi_{k, b} & =-\frac{2 \pi}{\lambda} r_{k} \sin \phi_{0} \sin \Psi_{k} \\
\varphi_{k, e} & =-\frac{2 \pi}{\lambda} r_{k} \cos \phi_{0} \cos \Psi_{k}
\end{aligned}
$$




\section{X.Lian et al.}

The array factor for the broadside array can be written as

$$
\begin{aligned}
& F_{\text {broadside }}(\phi)=\frac{1}{K} \sum_{k=1}^{K} \exp \left(j \frac{2 \pi}{\lambda} r_{k} \sin \Psi_{k} \sin \phi+\varphi_{k, b}\right) \\
& =\frac{1}{K} \sum_{k=1}^{K} \exp \left[j \frac{2 \pi}{\lambda} r_{k}\left(\sin \Psi_{k} \sin \phi-\sin \phi_{0} \sin \Psi_{k}\right)\right] \\
& =\frac{1}{K} \sum_{k=1}^{K} \exp \left[j 2 \pi \widetilde{R}\left(\sin \phi-\sin \phi_{0}\right) \frac{r_{k}}{R} \sin \Psi_{k}\right]
\end{aligned}
$$

Using the pdf of $z_{k}$ in equation (10), and the results in equations (10)-(13), we can derive in a similar way that

$$
\bar{P}_{\text {broadside }}(\phi)=E\left[|F(\phi)|^{2}\right]=\frac{1}{K}+\left(1-\frac{1}{K}\right) \mu_{b}(\phi)
$$

where

$$
\begin{gathered}
\mu_{b}(\phi)=\left|\frac{2 J_{1}\left(\alpha_{b}(\phi)\right)}{\alpha_{b}(\phi)}\right| \\
\alpha_{b}(\phi)=2 \pi \widetilde{R}\left(\sin \phi-\sin \phi_{0}\right)
\end{gathered}
$$

Similarly we can also obtain the average beampattern for the end-fire array, which can be written as

$$
\bar{P}_{\text {end-fire }}(\phi)=\frac{1}{K}+\left(1-\frac{1}{K}\right) \mu_{e}^{2}(\phi)
$$

where

$$
\begin{gathered}
\mu_{e}(\phi)=\left|\frac{2 J_{1}\left(\alpha_{e}(\phi)\right)}{\alpha_{e}(\phi)}\right| \\
\alpha_{e}(\phi)=2 \pi \widetilde{R}\left(\cos \phi-\cos \phi_{0}\right)
\end{gathered}
$$

The above equations (A-7)-(A-12) show the result of equations (16)-(21). 


\section{Biographies}

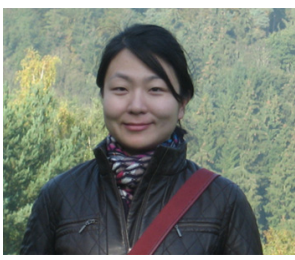

Xiaohua Lian was born on May 101980 in Urmqi, P.R. China. She received her Bachelor and Master of Engineering respectively in June 2002 and April 2005 from Nanjing University of Aeronautics and Astronautics, China. In Oct. 2013 she received her PhD degree from Delft University of Technology, The Netherlands. Her areas of interest include Smart antennas, Beamforming and cognitive radio.

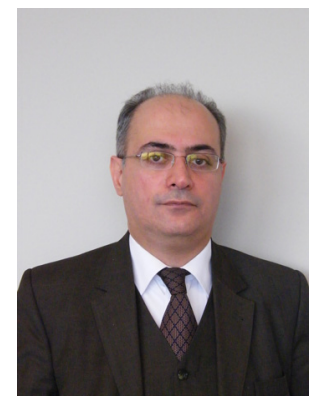

Homayoun Nikookar is an Associate Professor in the Microwave Sensing Systems and Signals Group of Faculty of Electrical Engineering, Mathematics and Computer Science at Delft University of Technology. He has received several paper awards at international conferences and symposiums and the 'Supervisor of the Year Award' at Delft University of Technology in 2010. He is the Secretary of the scientific society on Communication, Navigation, Sensing and Services (CONASENSE). He has published more than 130 refereed journal and conference papers, coauthored a textbook on 'Introduction to Ultra 
Wideband for Wireless Communications', Springer 2009, and has authored the book 'Wavelet Radio', Cambridge University Press, 2013.

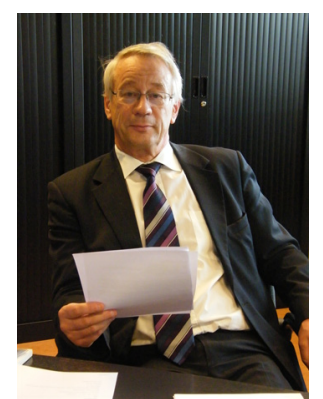

Leo P. Ligthart was born in Rotterdam, the Netherlands, on September 15, 1946. He received an Engineer's degree (cum laude) and a Doctor of Technology degree from Delft University of Technology in 1969 and 1985, respectively. He received honorary Doctorates from Moscow State Technical University of Civil Aviation in 1999, Tomsk State University of Control Systems and Radioelectronics in 2001 and Military Technical Academy, Romania in 2010. He is an academician of the Russian Academy of Transport. From 1992 to 2010 he held the chair of Microwave Transmission, Radar and Remote Sensing in the Faculty of Electrical Engineering, Mathematics and Computer Science, Delft University of Technology. In 1994, he founded the International Research Center for Telecommunications and Radar (IRCTR) and was the director of IRCTR for more than 16 years. His principal areas of specialization include antennas and propagation, radar and remote sensing, but he has also been active in satellite, mobile and radio communications. He has published over 600 papers and book chapters, and 2 books. Prof. Ligthart is a Fellow of the IET, IEEE and is the Chairman of the CONASENSE Foundation. 